International Journal of Pure and Applied Mathematics

Volume 109 No. 2 2016, 477-485

ISSN: 1311-8080 (printed version); ISSN: 1314-3395 (on-line version)

url: http://www.ijpam.eu

doi: 10.12732/ijpam.v109i2.21

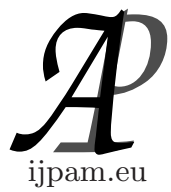

\title{
SPACE OCCUPYING MATHEMATICAL POINTS
}

\author{
Hector Cristino Estepan \\ 426 Highland Ave SW \\ Lenoir, NC 28645, USA
}

\begin{abstract}
Outlining a continuous mathematical vector function, a moving mathematical point, through a vector cross product, creates torques causing point rotation. Crossing the moving vector function, the point's velocity, with the rotational vector, the magnetic field, creates a charge, the electric field. Repulsing charges create a dynamic mathematical matrix, an aether.
\end{abstract}

AMS Subject Classification: 70E17, 26B12

Key Words: real mathematical point, vectors, motion, photon, cosmic microwave background radiation, fine structure constant, Maxwell's electromagnetic equations, charge, electricity, magnetism, rotation

Using the 23 century old Euclid's point definition, "that which has no part", mathematicians describe nature's reality with an unreal mathematical point. Unfolding nature, the moving space occupying mathematical point explores nature's reality as the point's motion develops the logical system requiring axioms.

1. The universe is space and non-space.

2. Space has no more than three orthogonal coordinates.

3. Non-space is a vector $X \mathbf{i}_{x}+Y \mathbf{i}_{y}+Z \mathbf{i}_{z}$.

4. The mathematical function's domain is $\mp \infty$.

Received: January 11, 2016

Published: September 12, 2016
(C) 2016 Academic Publications, Ltd.

url: www.acadpubl.eu 
Propose: The mathematical point:

1. is a sphere of radius $r_{o}$

2. has the constant speed $v_{O}$

3. has zero mass

4. has zero charge

Equating a point to the mathematical point, reality asserts:

1. A point occupies one space and time.

2. A point occupies its volume of space.

3. Two points cannot occupy the same space and time.

4. Constant speed points create a uniform coordinate system.

5. A moving point traces a continuous mathematical vector function.

6. A moving point cannot be created or destroyed.

7. Point collisions are elastic.

8. Two colliding points remain in the same plane.

9. The point's spherical diameter is the line's and plane's thickness.

10. Space and time are equivalent.

Proof. Length is a spatial dimension $l$.

Speed is length/time, or $l / t=v_{o}$, therefore $l=v_{o} t$ or, distance and time are equivalent.

In Cartesian coordinates, a point A outlining a mathematical vector function is

$$
A v_{o} \mathbf{i}_{x}
$$

where $A$ is the sphere of radius $r_{o}$ traveling with the constant speed $v_{o}$ in the positive $\mathrm{x}$-axis direction. The Cartesian coordinates has three independent vectors $A v_{o} \mathbf{i}_{x}, A v_{o} \mathbf{i}_{y}$, and $A v_{o} \mathbf{i}_{z}$.

Equating two intersecting vector functions, $A v_{o} \mathbf{i}_{\mathbf{x}}$ and $B v_{o} \mathbf{i}_{\mathbf{y}}$, to two point collision $A$, and $B$, mathematics has function $1, A v_{o} \mathbf{i}_{x}$, intersecting function 2 , $B v_{o} \mathbf{i}_{y}$ and reasons the result $A v_{o} \mathbf{i}_{y}$, and $B v_{o} \mathbf{i}_{x}$, or 
Rule 1. Exchanging direction, momentum, and energy, two colliding mathematical points maintain continuous functions.

While this rule determines each point's path, the rule limits collision to only two points.

Rule 2. Determining post collision position is impossible for more than two colliding points.

Proof. Pre collision: let point A outline line 1, point B outline line 2, and point $\mathrm{C}$ outline line 3 , then

post collision: Point A may outline line 2 or 3 , point B may outline line 1 or 3 , and point $\mathrm{C}$ may outline line 1 or 2 , therefore post collisions has ambiguous point position.

Two point collision preserves function continuity.

With constant speed, constant radius, and zero mass, colliding points cannot impart mechanical energy, $1 / 2 m v_{o}^{2}=0$, or mechanical momentum, $m v_{o}=$ 0 , to each other, and can only interchange directions creating new vectors in the same plane. It is impossible for two point collision to yield a three dimensional universe.

Rule 3. The mathematical universe is planar.

The expression $A v_{o} \mathbf{i}_{x}$ has the space occupying sphere $A$ with a radius $r_{o}$, whereas the non space occupying $v_{o} \mathbf{i}_{x}$ is an abstract mathematical representation of a previous event imparting constant speed to $A$, and opens the portals to Newton's first law of motion keeping the sphere in perpetual motion. With it's infinite number of static vectors, $X \mathbf{i}_{x}+Y \mathbf{i}_{y}+Z \mathbf{i}_{z}$, point $A$, a sphere of radius $r_{o}$, has non zero $X, Y$, and $Z$, otherwise $A$ ceases to be three dimensional.

Analysis: The object $A$, a sphere of radius $r_{o}$, has a velocity vector $v_{o} \mathbf{i}_{\mathbf{x}}$.

All objects, including sphere $A$, are static vector $X \mathbf{i}_{x}+Y \mathbf{i}_{y}+Z \mathbf{i}_{z}$, originating from the objec's center.

The cross product

$$
\left[v_{o} \mathbf{i}_{x}\right] \mathbf{x}\left[X \mathbf{i}_{x}+Y \mathbf{i}_{y}+Z \mathbf{i}_{z}\right]=v_{o}\left[-Z \mathbf{i}_{y}+Y \mathbf{i}_{z}\right],
$$

rotates the object with a torque proportional to the point's speed.

If $X=x / 2, Y=y / 2$, and $Z=z / 2$, the curl of equation (2) is $-v_{o} \mathbf{i}_{x}$, which is a velocity opposite to the sphere's velocity, and, if true, renders a motionless point. An equal and opposite motion renders a motionless object. Since the point is moving in the positive x-axis direction, the point's linear 
motion resistance must be less than the point's rotational resistance. Any point motion creates a rotational vector having a curl opposing the motion. Creating compatibility between Newton's first and third laws of motion, the point's motion explains Lenz's electromagnetic law.

Rotating point $A$, the dynamic vector $v_{o} \mathbf{i}_{x}$ crosses with the dynamic rotational vector $\mathbf{B}$, which is $\left(v_{o} / 2\right)\left[-z \mathbf{i}_{y}+y \mathbf{i}_{z}\right]$, to yield the cross product

$$
v_{o} \mathbf{i}_{x} \mathbf{x}\left(v_{o} / 2\right)\left[-z \mathbf{i}_{y}+y \mathbf{i}_{z}\right]=\mathbf{E}=\left(v_{o}^{2} / 2\right)\left[-y \mathbf{i}_{y}-z \mathbf{i}_{z}\right],
$$

which is a dynamic vector normal to the spherical surface, an electric charge. Also the cross product $v_{o} \mathbf{i}_{x} \mathbf{x}\left(v_{o}^{2} / 2\right)\left[-y \mathbf{i}_{y}-z \mathbf{i}_{z}\right]$ has the opposite direction and a different magnitude from $\mathbf{B}$. Mathematically, the point in motion generates

$$
\begin{aligned}
& \mathbf{E}=\mathbf{v} \times \mathbf{B}, \\
& \mathbf{B}=\mathbf{v} \times \mathbf{E},
\end{aligned}
$$

and

$$
\mathbf{v}=\mathbf{E x B}
$$

Because $A$ is anything occupying space, then $A$ can be a photon, an electron, a proton, a neutron, an atom, a compound, a molecule, a cell, a virus, a bacteria, a red blood cell, a plant, a fish, a bird, a cat, a dog, a woman, a man, a car, a boat, a plane, a rocket-ship, an asteroid, a moon, a planet, a star, a nebula, a galaxy, and the universe. Any moving space occupying object adheres to equations (4), (5), and (6).

Expressing the constant velocity vector $\mathbf{v}$ as

$$
\mathbf{v}=v_{o x} \mathbf{i}_{x}+v_{o y} \mathbf{i}_{y}+v_{o z} \mathbf{i}_{z}
$$

leads to

$$
v_{o x}=\partial x / \partial t, \quad v_{o y}=\partial y / \partial t, \quad v_{o z}=\partial z / \partial t .
$$

Given the vector operator

$$
\nabla=\mathbf{i}_{x} \partial / \partial x+\mathbf{i}_{y} \partial / \partial y+\mathbf{i}_{z} \partial / \partial z,
$$

then

$$
\nabla \times \mathbf{E}=\nabla \times[\mathbf{v x B}]=[[\nabla \cdot \mathbf{B}+\mathbf{B} \cdot \nabla] \mathbf{v}-[\nabla \cdot \mathbf{v}+\mathbf{v} \cdot \nabla] \mathbf{B}],
$$

and analysis of this result yields

$$
[\nabla \cdot \mathbf{B}]=0
$$


or the divergence of the vector $\mathbf{B}$ is zero.

Because the velocity $\mathbf{v}$ is constant, then

$$
[\mathbf{B} \cdot \nabla] \mathbf{v}=0,
$$

and

$$
\nabla \cdot \mathbf{v}=0
$$

leaving the term

$$
-[\mathbf{v} \cdot \nabla] \mathbf{B} .
$$

The term

$$
\mathbf{v} \cdot \nabla=v_{o x} \partial / \partial x+v_{o y} \partial / \partial y+v_{o z} \partial / \partial z
$$

from equation (8) becomes

$$
3 \partial / \partial t
$$

or expression (14) becomes

$$
-3 \partial \mathbf{B} / \partial t
$$

or

$$
\nabla \times \mathbf{E}=-3 \partial \mathbf{B} / \partial t .
$$

Similarly

$$
\nabla \times \mathbf{B}=[\nabla \cdot \mathbf{E}] \mathbf{v}-3 \partial \mathbf{E} / \partial t .
$$

These equations (19) and (18) are Maxwell's electromagnetic equations, but the derivation is strictly from mechanical considerations, and applicable to every moving object in the universe. There are two essential constraints:

1. The object must occupy space.

2. The object must have a constant speed.

If the speed is not constant, the equations are not as elegant, more complex, and bears little resemblance to Maxwell's equations.

Equations (4): $\mathbf{E}=\mathbf{v x B}$ and (5): $\mathbf{B}=\mathbf{v x E}$ yields

$$
\mathbf{E}^{\prime}=\left(1-v^{2} / c^{2}\right) \mathbf{E},
$$

where $\mathbf{E}^{\prime}$ is the spherical surface with lines normal to the surface coming in or going out, or respectively negative, or positive polarity, $v$ is the speed of the moving point, $c$ is a constant having the dimensions of speed, and $\mathbf{E}$ is the 
spherical surface with negative or positive polarity. Equation (20) demonstrates that the speed $v$ influences the polarity of $\mathbf{E}^{\prime}$ with respect to $\mathbf{E}$ 's polarity.

For $v$ less than $c, \mathbf{E}^{\prime}$ and $\mathbf{E}$ have the same polarity.

For $v$ greater than $c, \mathbf{E}^{\prime}$ and $\mathbf{E}$ have opposite polarities..

For $v$ equal to $c, \mathbf{E}^{\prime}$ has no polarity irrespective of $\mathbf{E}$ 's polarity.

For a neutral object, the speed determines object polarity, but an electron traveling at the speed $c$ has no charge, and traveling faster than $c$, has a positive charge. Similarly a proton traveling at a speed less than $c$ is a proton, but at faster than $c$ it is an electron. At speed $c$, all objects are neutral and explain the negative electron not crashing into the positive nucleus, obviating the quantum mechanics regions forbidding classical physics laws.

Equation (20) is Lorentz equation,

$$
\mathbf{F}=q[\mathbf{E}+\mathbf{v x B}]
$$

if $\mathbf{F} / q=\mathbf{E}^{\prime}$, but devoid of any electromagnetic considerations, and solely dependent on classical vector mechanics analysis.

Having points that cannot be created or destroyed, the mathematical universe has an infinite number of lines, and has only two point collision creating mathematical functions. Limiting the motion of these two points to the $\mathrm{x}$ and y directions does not guarantee two points collision, but separating these four different directions can create two point collision domains. Since the only function is the straight line, then there are four sets of straight lines; the set of points at

1. negative infinity traveling in the positive $\mathrm{x}$-axis direction,

2. negative infinity traveling in the positive $y$-axis direction,

3. positive infinity traveling in the negative $\mathrm{x}$-axis direction, and

4. positive infinity traveling in the negative $y$-axis direction.

Each point is a sphere of radius $r_{o}$, having the same speed $v_{o}$, and the same charge. With like charge repulsion, each mathematical point seeks the farthest distance from every point, but a repulsive charge universe is a dynamic matrix with each dynamic charge a constant distance $d$ from its 6 adjacent dynamic neighbors. The universal constant distance $d$ does not vary from point to point.

The collision between the set of points at negative infinity traveling in the positive $\mathrm{x}$-axis direction with the set of points at negative infinity traveling in the positive $y$-axis direction results in each point outlining a staircase function, which a 45 degrees clockwise rotation yields a 45 degree right triangular 
wave having the hypotenuse on the $\mathrm{x}$-axis, and the 90 degree vertex pointing in the positive y-axis direction. The two equal sides have length $d$, and the hypotenuse has length $\sqrt{2} d$, which is the wave's wavelength. The speed of the point along the 45 degree sides is $v_{o}$, while the 90 degree vertex speed, which is the propagating wave speed $v_{o} / \sqrt{2}$.

The dynamic matrix is a medium permitting the point's linear motion, permittivity $\left(\epsilon_{o}\right)$, and permeates the point's universal rotation, permeability $\left(\mu_{o}\right)$. These two constants along with $d$, and $v_{o}$ are universal constants, whereas mathematical analysis derives the propagating wave's speed, which depends on the two orthogonal resistances $\epsilon_{o}$, and $\mu_{o}$. Traveling at the constant propagating speed in the positive $\mathrm{x}$-axis direction, the transverse wave has a positive displacement $\mathbf{E}$ on the y-axis, and a positive displacement $\mathbf{B}$ on the $\mathrm{z}$-axis. Because the moving point creates both displacements, the displacements vary with time.

Molecular collision causes not only heat energy, but expresses energy as an equivalent temperature in the equation

$$
E=k T,
$$

where $E$ is heat energy, $k$ is constant, and $T$ is absolute temperature in degrees Kelvin. Creating heat energy, $E=k T$, the point collision generate the mathematical triangular waves, which occur with precise regularity, leading to a precise constant energy with $k$ a fundamental constant. Within the wavelength there is a fixed number of point collisions, so that heat energy is

$$
E=h f,
$$

where $E$ is heat energy, $h$ is constant, and $f$ is wave frequency. It follows that $T$ and $f$ are equivalent. In linear motion, the point has no energy, but, deviation from linear motion, according to Newton's first and second laws of motion, requires energy. Hence a point outlining a circle requires energy in spite of the fact that there is no detectable collision. The greater the point's orbital speed, the greater the energy $E=h f$.

Point collision in this dynamic matrix, where the distance $d$ tethers each dynamic point to its six adjacent dynamic neighbors, cause each point to vibrate about the distance $d$, and, similar to a plucked violin string, creates harmonics, which are discrete frequencies bounded by the diameter of the point, which sequentially goes through each harmonic frequency to dynamically rest at distance $d$.

In this ocean of mathematical dynamic points, the point's movement is similar to a tossed stone onto a quiet pond resulting in surface concentric waves 
having a height, or displacement of

$$
H=V / \pi r^{2},
$$

where $H$ is wave's height or displacement, $V$ is point's volume, and $r$ is distance from the point's center. Any object moving through any medium has the wave's displacement following the inverse square law. Sequentially oscillating at discrete harmonics, the point outlines sinusoidal mathematical functions, and transmits these movements with the inverse square law magnitude to all other dynamic matrix points. The medium's resistances, $\epsilon$, and $\mu$, determine the wave's propagating speed.

A point outlines each harmonic sinusoid transmitting the sinusoid's magnitude and frequency to all the other matrix points, and cannot create or destroy points. The point transmits the magnitude and frequency through the dynamic matrix medium. The zero mass point has no energy, the frequency is the point's directional change, which creates mechanical energy, and offers a different interpretation of the heat radiation equation. The discrete energy packets are the discrete harmonic frequencies, which is one point sequentially going through all the harmonics. The point's volume sinusoidal oscillations transmits the sinusoid with the speed $c$, a frequency $f$, the harmonic frequency, and an inverse square law magnitude, to all the other points in the mathematical universe.

The mathematical universe is theoretical, however, with the advent of cosmic microwave background radiation, CMB, in $1964^{1,2,3}$, evidence links the CMB to the theoretical mathematical point. The CMB are photons, which physics defines as particles having the constant speed $c$, having no mass and no charge ${ }^{4}$. Having a uniform distribution ${ }^{5}$ in the universe, the CMB has a quantitative homogeneous distribution of about $400 \mathrm{CMB}$ per cubic centimeter yielding 7.368 photons per $\mathrm{cm}$, and a distance of $1.357 \mathrm{~mm}$ between its 6 adjacent neighbors. The digits 1.357 are close to 1.37 , which are the digits of the fine structure constant. Letting the fine structure constant digits be the universal distance between photons gives a distribution of 388.9 photons per cubic cm. Taking $1.37 \mathrm{~mm}$ as the universal distance, makes the CMB's wavelength $(\sqrt{2}) 1.37=1.937 \mathrm{~mm}$, the experimental $\mathrm{CMB}$ wavelength is $1.9 \mathrm{~mm}$, which corresponds to the universal CMB temperature of 2.725 degrees Kelvin in Planck's heat radiation equation. Although the theoretical and experimental results are close, physics maintains photon appearance and disappearance, forbids mass to travel at the speed of light, and adheres to photons obeying uncertainty, which is the antithesis of the mathematical universe.

In summary, considering the mathematical point as a space occupying object having zero mass, zero charge, and constant speed, the analysis results in 
continuous mathematical functions with the point having no birth and no death. The point's dimension is a vector expression, and through the point's motion and vector product, causes point rotation. The vector product of the motion vector, $\mathbf{v}$ and the rotational vector $\mathbf{B}$ yields $\mathbf{E}$, and easily derives Maxwell's electromagnetic laws linking Newton's mechanics to Maxwell's electromagnetism linking it to light. But gigantic principles, the photons being created and destroyed, mass cannot travel at speed c or greater, and the uncertainty principle, stand in the way of the photon's link to the mechanical mathematical point. These principles have to disappear to bring life to the mathematical point. Lorentz's equation shows that speed above $c$ change the particle's polarity, and speed below $c$ maintains the particle's polarity, but at speed $c$ there is no polarity. Suspending electromagnetic interaction, the point speed $c$ explains the forbidden atom domains. Colliding points generate heat and transverse waves, linking the mechanical heat production and wave's frequency. Finally, having a speed greater than $c$, the point, a charged particle, creates a dynamic matrix having a fine structure constant digit's distance between points, creates transverse waves propagating at speed $c$, with magnetic and electric displacements within their respective resistances, permeability and permittivity. The resemblance between the findings of this mathematical dynamic matrix and the CMB offers evidence for the ether's existence. The mathematical point formulation deserves further exploration in more capable hands, but nevertheless, the mathematical analysis stands on its own, although the novice author's interpretation may be lacking.

\section{References}

[1] https://en.wikipedia.org/wiki/Cosmic microwave background radiation

[2] http://aether.lbl.gov/www/science/cmb.html

[3] https://books.google.com/books?isbn=1139855565

[4] https://en.wikipedia.org/wiki/Photon

[5] background.uchicago.edu/ whu/ 
